\title{
Inertial and stick-slip regimes of unstable adhesive tape peeling
}

\author{
Marie-Julie Dalbe, ${ }^{\text {ab }}$ Richard Villey, ${ }^{c d}$ Matteo Ciccotti, ${ }^{d}$ Stéphane Santucci, ${ }^{* a}$ \\ Pierre-Philippe Cortet ${ }^{\star c}$ and Loïc Vanel ${ }^{\mathrm{b}}$
}

\begin{abstract}
We present an experimental characterization of the detachment front unstable dynamics observed during the peeling of pressure sensitive adhesives. We use an experimental set-up specifically designed to control the peeling angle $\theta$ and the peeled tape length $L$, while peeling an adhesive tape from a flat substrate at a constant driving velocity $V$. High-speed imaging allows us to report the evolution of the period and amplitude of the front oscillations, as well as the relative durations of their fast and slow phases, as a function of the control parameters $V, L$ and $\theta$. Our study shows that, as the driving velocity or the peeling angle increases, the oscillations of the peeling front progressively evolve from genuine "stick-slip" oscillations, made of alternating long stick phases and very brief slip phases, to sinusoidal oscillations of amplitude twice the peeling velocity. We propose a model which, taking into account the peeling angle-dependent kinetic energy cost to accelerate and decelerate the peeled tape, explains the transition from the "stick-slip" to the "inertial" regime of the dynamical instability. Using independent direct measurements of the effective fracture energy of the adhesive-substrate joint, we show that our model quantitatively accounts for the two regimes of the unstable dynamics.
\end{abstract}

\section{Introduction}

When peeling a pressure sensitive adhesive from its substrate at a constant velocity $V$, the propagation of the detachment front can display periodic oscillations between fast and slow phases. This "stick-slip" peeling instability - historically revealed by measurements of the associated oscillations of the peeling force $^{1-7}-$ is due to the decrease of the effective fracture energy $\Gamma\left(v_{\mathrm{p}}\right)$ of the adhesive-substrate joint over a finite range of detachment front velocity $v_{\mathrm{p}} \cdot{ }^{1-5,7-11}$ Such a decrease has been proposed to be the consequence of a complex coupling between the rheology of the adhesive material and its confinement in a thin layer. ${ }^{12-18}$ The relevant ingredients to model $\Gamma\left(v_{\mathrm{p}}\right)$ however remain a debated issue and no definitive theory has been found yet (see discussion in ref. 18).

During a peeling experiment, the elasticity (most often due to the peeled ribbon) between the detachment front and the point at which the constant driving velocity $V$ is imposed provides a degree of freedom to the system, namely the detachment front position. For driving velocities in the decreasing range of the fracture energy $\Gamma$, the peeling front dynamics eventually becomes unstable. A small forward fluctuation of the front position with respect to the steady propagation makes the force in the peeled tape decrease, which, because of the fracture energy decreasing with the front velocity, makes the front accelerate so that the fluctuation is amplified.

In the mid 1980's, Maugis and Barquins ${ }^{8,9}$ proposed a quasistatic model to describe the limit cycles of this instability. The front dynamics is assumed to be still described by the steady state equation, i.e. an equilibrium between the energy release rate of the system $G$ and the effective fracture energy $\Gamma$. This amounts to consider that the changes in the front velocity take place slowly enough for the peeling to remain quasi-static. In this model, the range of velocities over which $\Gamma$ is decreasing is however forbidden to the instantaneous detachment front velocity $v_{\mathrm{p}}$, which alternates regularly between quasi-static phases in the small velocity stable domain and quasi-static phases in the large velocity stable domain, with velocity jumps between the two.

Coupling their model to direct measurements of the two stable and increasing branches of the effective fracture energy $\Gamma\left(v_{\mathrm{p}}\right)$, Maugis and Barquins succeeded in predicting the period of the stick-slip instability in quantitative agreement with measurements ${ }^{9}$ for a standard adhesive tape peeled directly from its roll. The experimental validation of their own model 
remained however limited to the lowest part of the unstable range of driving velocities, since they did not succeed in measuring the instability period at larger unstable driving velocities (typically of the order of the meter per second).

Dalbe and coworkers ${ }^{19}$ (all authors of the present article) reproduced the peeling experiments of Maugis and Barquins ${ }^{9}$ for a similar adhesive tape. They were able, using a fast camera, to capture the detachment front dynamics at larger unstable driving velocities (however still not covering the whole unstable domain). This study revealed that, at larger driving velocities $V$, the instability period tends towards a lower bound independent of $V$, a behavior which departs from the prediction of the quasistatic model. These results were proposed to reflect the growing role, as $V$ increases, of some inertial effects in the selection of the limit cycles. At even larger driving velocities, ${ }^{21}$ the adhesive tape roller, which is mounted on a free pulley, develops pendular oscillations. This motion of the roller led to strong and slow oscillations of the peeling angle $\theta$ and revealed the strong influence of $\theta$ on the instability.

In order to overcome these experimental limitations, we have developed a set-up that allows to control both the peeling angle $\theta$ and the driving velocity $V$ and also to suppress possible inertial effects related to the substrate motion (the rotation of the roller in the previous experiments). This system, which is the one considered here, allowed to confirm the influence of the peeling angle $\theta$ on the domain of unstable velocities, which broadens as $\theta$ decreases. ${ }^{22}$ This study also revealed that, as $V$ or $\theta$ increases, the limit cycles of the instability progressively change from true "stick-slip" relaxation oscillations, made of a long stick phase and a very brief slip phase, to sinusoidal oscillations of amplitude twice the driving velocity $V$.

In the present manuscript, we provide a thorough experimental characterization of the transition between the two different unstable peeling regimes for adhesive tapes. We report the evolution of the instability period and amplitude and of the relative duration of the fast and slow phases of the oscillations as a function of the three control parameters, which are the peeling angle $\theta$, the peeling velocity $V$ and the peeled tape length $L$ between the detachment front and the pulling point. As reported previously, ${ }^{19}$ inertial effects move the instability away from quasi-static "stick-slip". It was suggested in ref. 22 that the peeling dynamics in the inertial regime is compatible with an equation of motion including an inertial term that involves the acceleration of the peeling front. The proposed equation, although already reported in the literature, is however purely empirical. Here, we propose a model which takes into account the energy cost to accelerate and decelerate the peeled tape and leads naturally to a similar equation. A peeling angle dependence of the effective inertia is predicted by the model, in agreement with experimental observations.

\section{Experimental set-up}

We use a commercial adhesive tape, $3 \mathrm{M}$ Scotch ${ }^{\circledR} 600$, already studied in ref. 18-24. It is made of a polyolefin blend backing (thickness $e=34 \pm 1.2 \mu \mathrm{m}$, width $b=19 \mathrm{~mm}$, tensile modulus $E=1.41 \pm 0.11 \mathrm{GPa}$ ) coated with a $20 \mu \mathrm{m}$ layer of synthetic acrylic adhesive of negligible rigidity. The experiments have been performed at a temperature of $22.3 \pm 0.9{ }^{\circ} \mathrm{C}$ and a relative humidity of $43 \pm 9 \%$.

The experimental set-up, described in more details in ref. 22, consists in a $3 \mathrm{~m}$ long bar translated at an imposed velocity $V$ thanks to a servo-motor (see Fig. 1). A first layer of adhesive tape applied on this bar is used as the substrate of a second layer of the same adhesive. The first adhesive tape layer used as a substrate is initially covered with an anti-adhesive release coating. After multiple peeling from the same substrate, this coating can be damaged, resulting in variations of the adhesion force for the same control parameters. To ensure repeatability, we have carefully removed the release coating using an ethanolsoaked wipe prior to our peeling experiments. In our experiments the adhesion is therefore larger than for experiments where the adhesive was directly peeled from the commercial roll, in which case the release coating is intact. ${ }^{21,23}$

During the bar translation, the upper adhesive tape is winded by a rotating motor at velocity $R \omega$, with $R$ the radius of the rotating axis and $\omega$ its rotation rate. The two motors are electronically enslaved so that, during an experiment, the winding and translation proceed at the same velocity $V=R \omega$ (including during transient acceleration and deceleration). Under these conditions, if the detachment front dynamics is steady, the peeled tape length $L$ and the peeling angle $\theta$ are constant (Fig. 1) and the front velocity is $v_{\mathrm{p}}(t)=V$.

On the contrary, when the peeling dynamics is unstable, the detachment front velocity $v_{\mathrm{p}}(t)$ oscillates periodically with an amplitude larger than or close to the driving velocity $V$ (see ref. 22). The real value of our setup is that, in this unstable regime, the oscillations of the peeling angle and of the peeled tape length remain small: among all the experiments presented

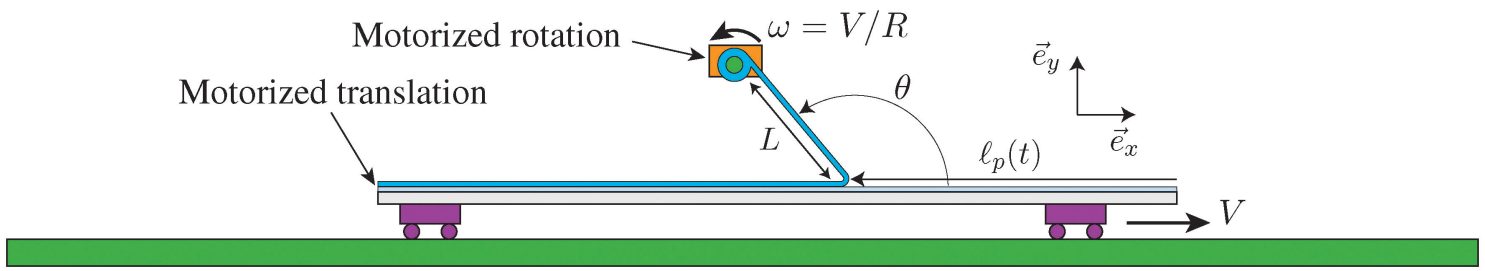

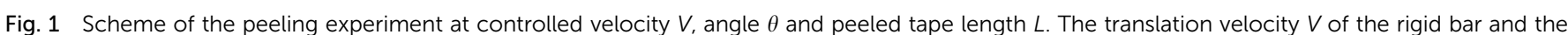

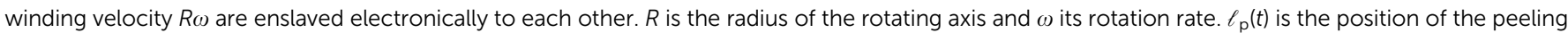
front with respect to the translated substrate. 
in this paper, they range from $0.1^{\circ}$ to $2^{\circ}$ and from $0.5 \%$ to $3 \%$ respectively. Note that the peeling front velocity $v_{\mathrm{p}}(t)$ follows precisely the imposed velocity $V$ once averaged over time scales larger than the period of the instability oscillations (ranging from a few to a few tens of ms).

We performed peeling experiments varying systematically the three relevant "control parameters": the driving velocity $0.03 \mathrm{~m} \mathrm{~s}^{-1} \leq V \leq 4.2 \mathrm{~m} \mathrm{~s}^{-1}$, the peeled tape length $20 \mathrm{~cm} \leq$ $L \leq 134 \mathrm{~cm}$ and the peeling angle $30^{\circ} \leq \theta \leq 150^{\circ}$. The two motors are able to accelerate and decelerate strongly enough so that a steady-state regime at constant velocity $V$ is achieved over at least $1 \mathrm{~m}$ of peeling.

During an experiment, we image the peeling point region thanks to a high speed camera (PHOTRON FASTCAM APX RS) with frame rates from 500 to 20000 fps depending on the peeling velocity. The corresponding images are made of $896 \times$ 512 to $384 \times 224$ pixels respectively which correspond to resolution in the range 40 to $80 \mu \mathrm{m}$ per pixel. From the recorded image time series, we extract the peeling point position thanks to a home made image processing. We detect the location $\ell_{\text {lab }}$ in the laboratory frame of the peeled tape at a small distance $(0.7 \pm$ $0.1 \mathrm{~mm}$, solid line in Fig. 2) from the substrate with a precision of about one pixel size, thanks to subpixel interpolation. The effective precision which we obtain on the peeled taped position leads to typical errors on the peeled tape velocity in the laboratory of about $\pm 1 \%$.

In parallel, we measure the instantaneous velocity of the substrate $\mathrm{d} \ell_{\text {bar }} / \mathrm{d} t$ through maximizing the cross-correlation, between shifted successive images, of a gray level line parallel to the substrate (dashed line in Fig. 2). This gray level line which has first been subpixel interpolated corresponds to the pattern of a riglet attached to the rigid bar. Through this method, we are able to measure the translation velocity of the substrate $\mathrm{d} \ell_{\mathrm{bar}} / \mathrm{d} t$ with a relative typical precision of $\pm 0.4 \%$.

From these two time series, $\ell_{\text {lab }}$ and $\mathrm{d} \ell_{\text {bar }} / \mathrm{d} t$, we finally compute the dynamics of the tape very close to the peeling front $\ell_{\mathrm{p}}(t)=\ell_{\text {lab }}+\ell_{\mathrm{bar}}$. From this estimate of the peeling front dynamics, we compute the front velocity relative to the substrate $v_{\mathrm{p}}(t)=\mathrm{d} \ell_{\mathrm{p}} / \mathrm{d} t=\mathrm{d} \ell_{\text {lab }} / \mathrm{d} t+\mathrm{d} \ell_{\text {bar }} / \mathrm{d} t$.

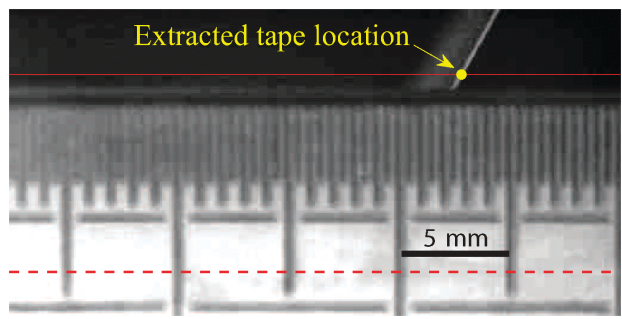

Fig. 2 Image recorded by the high speed camera during an experiment with $\theta=60^{\circ}, L=50 \mathrm{~cm}$ and $V=1 \mathrm{~m} \mathrm{~s}^{-1}$. From this image, we extract a line of gray levels parallel to the substrate (solid line) at a distance of $0.7 \pm$ $0.1 \mathrm{~mm}$. After a subpixel interpolation, we estimate the location of the peeling front from the maximum of the gray levels. The gray levels of a riglet attached to the bar (along the dashed line) is used to measure the velocity of the rigid substrate thanks to cross-correlations between successive shifted images.

\section{Limit cycles of the instability}

For each experiment, we compute the peeling front velocity time series $v_{\mathrm{p}}(t)=\mathrm{d} \ell_{\mathrm{p}}(t) / \mathrm{d} t$. As reported in Dalbe et al., ${ }^{22}$ the peeling dynamics is unstable over a finite range of driving velocities $V_{\mathrm{a}}(\theta)<V<V_{\mathrm{d}}(\theta)$ which gets smaller as the peeling angle $\theta$ increases [for the adhesive-substrate joint studied here, $V_{\mathrm{a}}(\theta)$ ranges typically from 1 to a few tens of $\mathrm{cm} \mathrm{s}^{-1}$ and $V_{\mathrm{d}}(\theta)$ from more than $4 \mathrm{~m} \mathrm{~s}^{-1}$ down to about $3 \mathrm{~m} \mathrm{~s}^{-1}$, for $\theta$ ranging from $30^{\circ}$ to $150^{\circ}$. This range does not significantly depend on the peeled tape length (for $20 \mathrm{~cm}<L<134 \mathrm{~cm}$ ). In this regime, regular periodic oscillations of $v_{\mathrm{p}}(t)$ are observed with an amplitude larger than or equal to $V$. In this section, we describe in details the temporal periodicity and shape of these velocity oscillations as a function of the control parameters $(V, \theta, L)$.

We divide the periodic velocity signal into oscillation cycles, arbitrarily choosing the beginning of the $n$th cycle at the first time $t_{n}\left(>t_{n-1}\right)$ such that $v_{\mathrm{p}}\left(t_{n}\right)=V$ and $\frac{\mathrm{d} v_{\mathrm{p}}}{\mathrm{d} t}\left(t_{n}\right)<0$ (see Fig. 4(a) or (b)). From this decomposition, we extract the duration of each oscillation cycle $t_{n+1}-t_{n}$ and the average period of the instability $T_{\mathrm{ss}}=\left\langle t_{n+1}-t_{n}\right\rangle_{n}$ over all cycles in the considered experiment (the number of cycles in one experiment ranges between 5 and 1000 depending on $(V, \theta, L)$ but is most of the time of the order of 100). The period of the oscillations is generally quite stable: the standard deviation of $t_{n+1}-t_{n}$ in one experiment ranges from $1 \%$ to $10 \%$ of $T_{\mathrm{ss}}$ over the range of studied parameters $(V, \theta, L)$.

For all studied peeling angles $\theta$ and driving velocities $V$, we observe that the oscillation period $T_{\mathrm{ss}}$ is nearly proportional to the peeled tape length $L$ as illustrated in Fig. 3 for $\theta=60^{\circ}$ (and several velocities $V$ ) and for $V=0.5 \mathrm{~m} \mathrm{~s}^{-1}$ (and several angles $\theta$ ). This result has already been evidenced for similar adhesive tapes for $\theta \simeq 90^{\circ}$ and small driving velocities by Barquins and Maugis $^{9}$ (namely for $V<0.6 \mathrm{~m} \mathrm{~s}^{-1}$ ) and Dalbe et al. ${ }^{19}$ (for $V<$ $1.5 \mathrm{~m} \mathrm{~s}^{-1}$ ) with experiments where peeling proceeds directly from the commercial tape roll. We extend here this result to the entire range of parameters $(V, \theta)$ for which the dynamical instability develops. ${ }^{22}$

We further consider the ratio $T_{\mathrm{ss}} / L$, nearly independent of the peeled tape length $L$, and average it over the different $L$ values. In Fig. 4(c), we report these data as a function of $V$ for the five studied peeling angles $\theta$. For the lowest peeling angle $\theta=30^{\circ}$, the cycle period decreases with the driving velocity as $T_{\mathrm{ss}} / L \propto 1 / V$, while it is nearly constant with $V$ at the largest peeling angles $\theta=120^{\circ}$ and $\theta=150^{\circ}$. For intermediate angles, both behaviors are observed: for low driving velocities $T_{\mathrm{ss}} / L \propto 1 / V$, while for large velocities $T_{\mathrm{ss}} / L$ saturates to a low value $1 / v_{\text {sat }}$ weakly dependent of the angle: $v_{\text {sat }}=220 \pm$ $70 \mathrm{~m} \mathrm{~s}^{-1}$. The velocity of transition between the two regimes rapidly decreases when the peeling angle $\theta$ increases. The behavior $T_{\mathrm{ss}} / L \propto 1 / V$ has also already been reported for $\theta \simeq$ $90^{\circ}$ by Barquins and Maugis ${ }^{9}$ and by Dalbe et al. ${ }^{19}$ in both cases for $V<0.6 \mathrm{~m} \mathrm{~s}^{-1}$ only, in agreement with the data shown here.

We now characterize the typical shape of the velocity oscillations in the two asymptotic regimes of the unstable peeling evidenced in Fig. 4(c). For each cycle in one experiment, we rescale the 

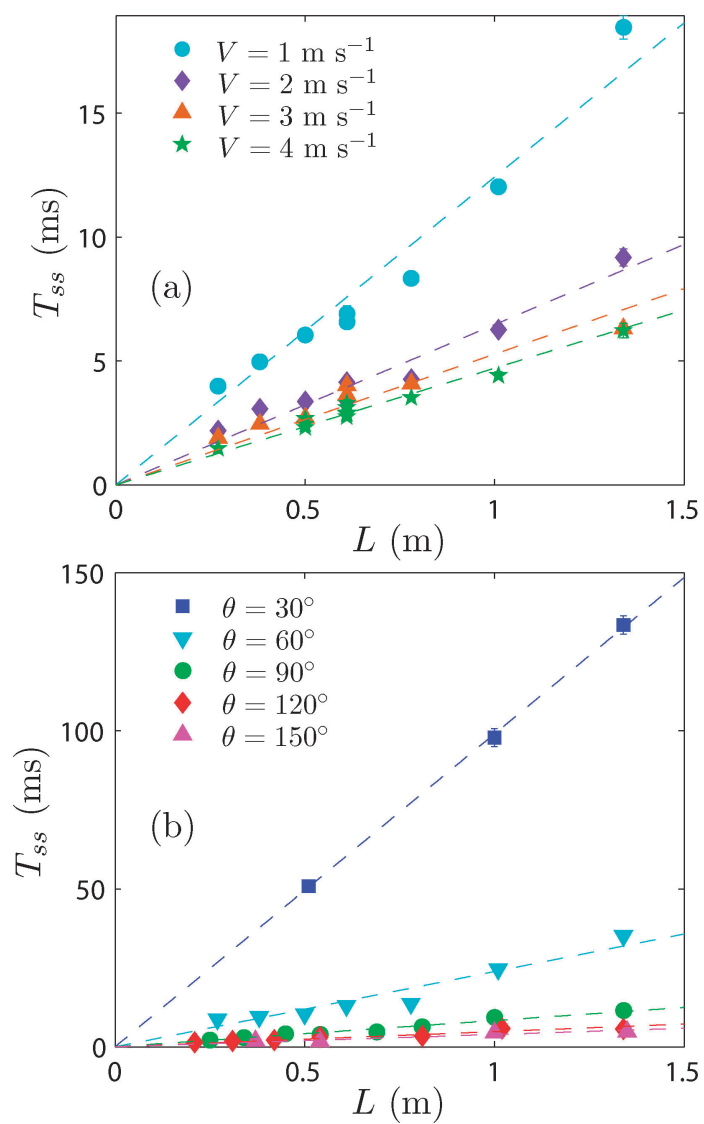

Fig. 3 Period $T_{\mathrm{ss}}$ of the instability oscillation as a function of the peeled tape length $L$ (a) for different imposed driving velocities $V$ and $\theta=60^{\circ}$ and (b) for different peeling angles $\theta$ and $V=0.5 \mathrm{~m} \mathrm{~s}^{-1}$. The dashed lines are linear fits. Each point represents the average over all cycles during one experiment.

time basis as $\varphi=\left(t-t_{n}\right) /\left(t_{n+1}-t_{n}\right) \in[0 ; 1]$. We further compute the phase averaged velocity cycle $\left\langle v_{\mathrm{p}}\right\rangle(\varphi)=\left\langle v_{\mathrm{p}}\left(t_{n}+\left(t_{n+1}-t_{n}\right) \varphi\right)\right\rangle_{n}$ over all the cycles during one experiment in order to get rid of fluctuations around the typical cycle. In Fig. 4(a) and (b), we show two typical examples of such velocity oscillations, corresponding to the regime where $T_{\mathrm{ss}} / L \propto 1 / V$ in (a) and to the regime where $T_{\mathrm{ss}} / L \simeq 1 / v_{\mathrm{sat}}$ in (b).

In Fig. 4(a) $\left(V=0.20 \mathrm{~m} \mathrm{~s}^{-1}, \theta=30^{\circ}, L=1.34 \mathrm{~m}\right)$, one can observe a long "stick" phase associated to a very low peeling front velocity followed by a very brief "slip" phase associated to large velocities (here about $33 \mathrm{~m} \mathrm{~s}^{-1}$ ): the stick phase represents $98 \%$ of the whole cycle duration here. Such a velocity cycle is archetypal of stick-slip relaxation oscillations as described by the quasi-static model of Barquins and Maugis. ${ }^{9}$ One can notice that for the peeling angle considered here, $\theta=30^{\circ}$, this quasi-static model predicts the correct scaling observed for the oscillation period, i.e. $T_{\mathrm{ss}} \propto L / V$. In Fig. 4(b), for an experiment well into the regime where $T_{\mathrm{ss}} / L$ is nearly constant $\left(V=1.50 \mathrm{~m} \mathrm{~s}^{-1}\right.$, $\left.\theta=150^{\circ}, L=0.54 \mathrm{~m}\right)$, the velocity oscillation is very different: it is quasi-sinusoidal (and thus fast and slow phases are of comparable durations) with an amplitude of about $2 \mathrm{~V}$. This regime of the instability can obviously not be considered as a stick-slip dynamics (there is no stick phase) and will further be referred to as the "inertial regime".
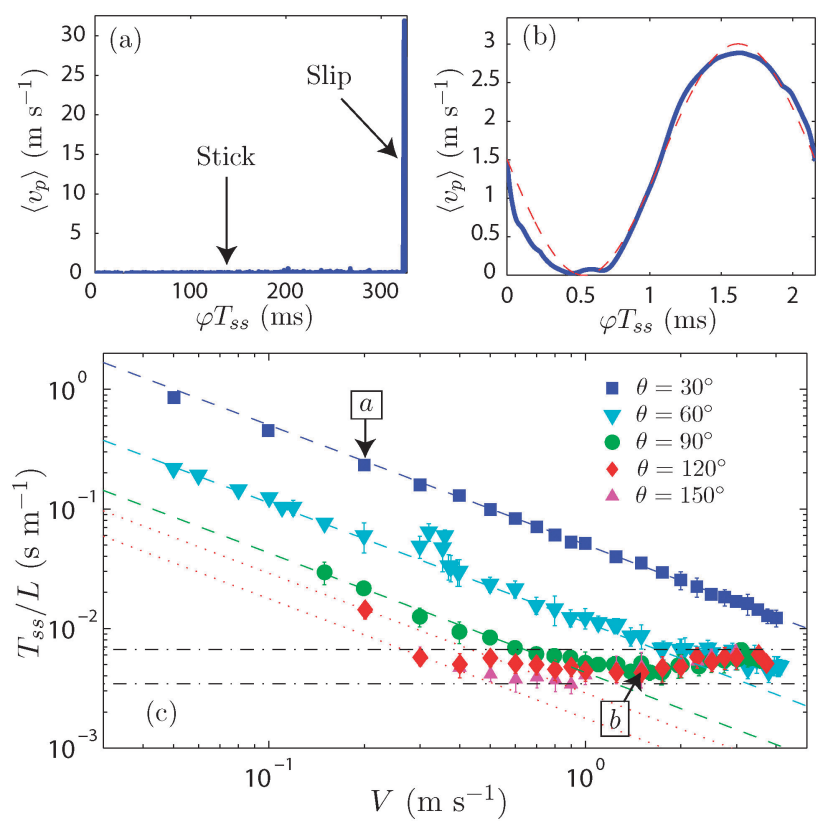

Fig. 4 (a and b) Phase averaged velocity cycle $\left\langle v_{p}\right\rangle$ as a function of $\varphi T_{\text {ss }}$ with $T_{\mathrm{ss}}$ the average period over the experiment and $\varphi$ the dimensionless time. In (a), $V=0.20 \mathrm{~m} \mathrm{~s}^{-1}, \theta=30^{\circ}, \mathrm{L}=1.34 \mathrm{~m}$; in (b), $V=1.50 \mathrm{~m} \mathrm{~s}^{-1}$ $\theta=150^{\circ}, L=0.54 \mathrm{~m} . \operatorname{In}(\mathrm{b})$, the red dashed line represents a sinusoidal oscillation of period $T_{\text {ss }}$ and amplitude $2 V$. (c) $T_{\text {ss }} / L$ as a function of $V$ for different peeling angles $\theta$. The experimental data are averaged over different lengths $L$. The three dashed lines represent best fits of the data for $\theta=30^{\circ}, 60^{\circ}$ and $90^{\circ}$ by prediction (14) of the quasi-static model, with $\Delta \Gamma$ as an adjustable parameter (only the subset of data where $T_{\text {ss }} / L$ is a clearly decreasing function of $V$ is considered for the fit). The two dotted lines also correspond to eqn (14) but are not fits. They represent the two extreme behaviors compatible with the data for $\theta=120^{\circ}$. The two horizontal dashed-dotted lines indicate the range of experimental values observed in the saturation regime, corresponding to $T_{\text {ss }} / L=$ $1 / v_{\text {sat }} \in 5.0 \pm 1.7 \mathrm{~ms} \mathrm{~m}^{-1}$.

We also report in Fig. 5 (reproduced from ref. 22) the peakto-peak amplitude $\Delta v_{\mathrm{p}}$ of the velocity oscillations as a function of the driving velocity $V$ for the different peeling angles $\theta$. This amplitude is nearly independent of the peeled tape length $L$

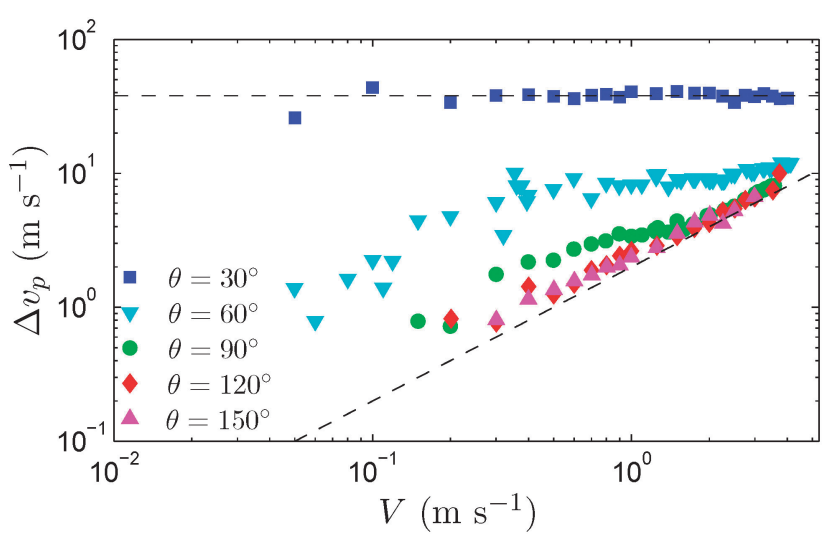

Fig. 5 Amplitude $\Delta v_{p}$ of the velocity oscillations as a function of the driving velocity $V$ for different peeling angles $\theta$ (figure reproduced from ref. 22). Each marker represents data averaged over different peeled tape lengths $L$. The lower dashed line is $\Delta v_{p}=2 V$ and the upper one $\Delta v_{p}=38 \mathrm{~m} \mathrm{~s}^{-1}$. 
(over the range studied here) and the data have therefore been averaged over $L$. At the smallest peeling angle $\theta=30^{\circ}$, for which the instability is always in the "stick-slip" regime, the oscillations amplitude is almost independent of the driving velocity, $\Delta v_{\mathrm{p}}=38 \pm 5 \mathrm{~m} \mathrm{~s}^{-1} . \Delta v_{\mathrm{p}}$ decreases with the peeling angle, this tendency being stronger as the driving velocity gets smaller. At large peeling angles, the oscillations amplitude saturates to a low value equal to twice the driving velocity, $\Delta v_{\mathrm{p}}=2 \mathrm{~V}$, in agreement with the quasi-sinusoidal cycle shown in Fig. 4(b). This scaling for $\Delta v_{\mathrm{p}}$ is observed in correlation with the saturation of the instability period $T_{\mathrm{ss}}$ to the constant value $L / v_{\text {sat }}$ it is therefore also typical of what we named the "inertial regime".

To characterize completely the velocity cycles of the instability, we finally consider the relative duration $R_{\mathrm{T}}=T_{\text {stick }} / T_{\mathrm{ss}}$ of the slow phase, during which $\left\langle v_{\mathrm{p}}\right\rangle<V$, with respect to the whole cycle period $T_{\mathrm{ss}}$ (in the stick-slip regime, this slow phase duration is very close to the stick-slip duration). These data are reported in Fig. 6 as a function of the driving velocity $V$ and for the five studied peeling angles $\theta$. Once again, each point corresponds to data averaged over the peeled tape length $L$, since $R_{\mathrm{T}}$ appears not to depend significantly on $L$ (over the range studied here). At the smallest peeling angle studied, $\theta=30^{\circ}, R_{\mathrm{T}}(V)$ linearly decreases from 1 to 0.86 for $V$ from $5 \mathrm{~cm} \mathrm{~s}^{-1}$ to $4 \mathrm{~m} \mathrm{~s}^{-1}$. This data confirms the stick-slip nature of the instability with a much longer duration spent at low velocities than at large velocities during a cycle. When the peeling angle $\theta$ increases at a given driving velocity, the relative duration of the slow phase (during which $\left\langle v_{\mathrm{p}}\right\rangle<V$ ) decreases while duration of the fast phase $\left(\left\langle v_{\mathrm{p}}\right\rangle>V\right)$ increases; the slow phase however always represents at least half of the cycle period $\left(R_{\mathrm{T}} \approx 0.5\right)$. At the largest peeling angles $\left(\theta=120^{\circ}\right.$ and $\left.\theta=150^{\circ}\right)$ and at large driving velocities, when the instability is well into the "inertial regime", we verify that the fast and slow phases durations become comparable $\left(R_{\mathrm{T}} \rightarrow 0.5\right)$ which is consistent with the sinusoidal shape of the oscillations illustrated in Fig. 4(b).

In the following, we propose a model that accounts for both the "stick-slip" and "inertial" regimes of the dynamical instability of the peeling of pressure sensitive adhesives.

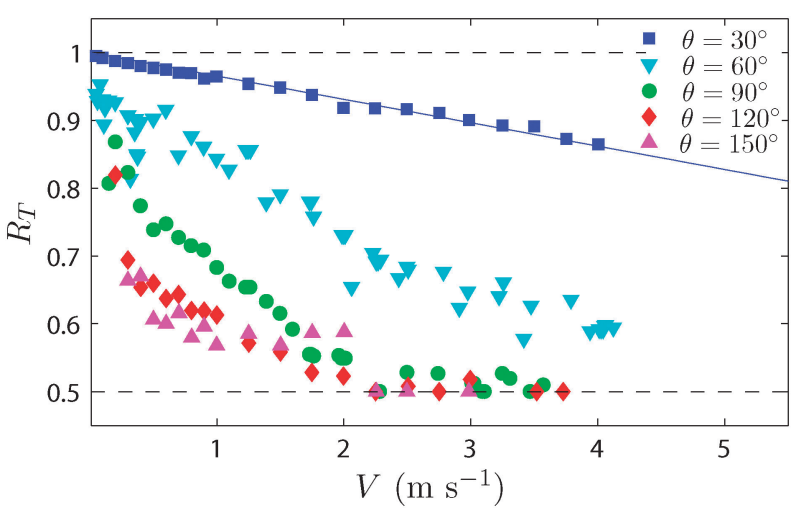

Fig. 6 Relative duration $R_{\mathrm{T}}=T_{\text {stick }} / T_{\text {ss }}$ of the slow phase, during which $\left\langle v_{\mathrm{p}}(t)\right\rangle<$ $V$, with respect to the whole cycle period $T_{\text {ss }}$ as a function of the driving velocity $\checkmark$ for different peeling angles $\theta$. Each marker represents data averaged over different peeled tape lengths $L$. The continuous line shows a best fit of the data at $\theta=30^{\circ}\left(R_{\mathrm{T}}=1-V / V_{\mathrm{m}}\right.$ from eqn (13)) leading to $V_{\mathrm{m}}=29 \pm 5 \mathrm{~m} \mathrm{~s}^{-1}$.

\section{A quasi-static model for any peeling angles}

\subsection{Fracture energy during peeling}

During a peeling experiment, when the detachment front of the adhesive-substrate joint propagates steadily at a velocity $v_{\mathrm{p}}$, the peeling dynamics can be described by a balance equation

$$
G=\Gamma\left(v_{\mathrm{p}}\right)
$$

between the mechanical energy release rate $G$ of the system and an effective fracture energy $\Gamma . G$ measures the release of mechanical energy per unit surface of fracture growth and $\Gamma$ accounts for the energy dissipation associated with the fracture advance, also per unit surface. In the peeling geometry, the mechanical energy release rate writes ${ }^{25}$

$$
G=\frac{F}{b}(1-\cos \theta)+\frac{F^{2}}{2 b^{2} e E}
$$

where $F$ is the force transmitted along the tape, $b$ the tape width, $e$ the tape backing thickness and $E$ its tensile modulus (the adhesive layer rigidity is negligible in comparison). The first rhs term in eqn (2) corresponds to the work done by the applied force $F(t)$ when peeling an inextensible tape, while the second rhs term accounts for the additional work of this force to elastically elongate the tape ligament detached when the fracture advances minus the elastic energy stored in this ligament, and not available for the fracture. According to eqn (1) and (2), when the peeling is steady, the measurement of the peeling force $F$ gives a direct access to the energy release rate $G$ and therefore to the peeling fracture energy $\Gamma$.

Using either a dynamical torquemeter (interface T2-2 Nm) coupled to the winding cylinder, in the experimental set-up presented in Section 2, or an Instron testing machine (model 3343 ) coupled to a motorized tilted translation stage (for $V \leq$ $1 \mathrm{~cm} \mathrm{~s}^{-1}$ ), we could measure the peeling force $F$ as a function of the imposed driving velocity $V$ for various peeling angles $\theta$ (see ref. 18 for experimental details). We report in Fig. 7 the corresponding energy release rates $G$.

At driving velocities smaller than $V_{\mathrm{a}}(\theta)$ (values reported in Table 1), the peeling is steady and $F, G$ and $\Gamma$ are slowly increasing functions of the driving velocity $V$ (equal to the instantaneous peeling front velocity $v_{\mathrm{p}}$ ). More precisely, we observe that $G$ follows a power law of $V$ with an exponent increasing from 0.05 to 0.10 for $\theta$ increasing from $30^{\circ}$ to $150^{\circ}$. More importantly, in this steady peeling regime, the energy release rate $G$ is an increasing function of $\theta$, in agreement with recent measurements. ${ }^{18}$ It is important to recall that in our experiments, the first adhesive layer used as a substrate is cleansed of its release coating, contrary to the experiments described in ref. 18, leading to larger values of adhesion ( $G$ is 3 to 6 times higher in this paper). In Villey et al. ${ }^{18}$ the observed increase of the fracture energy $\Gamma(\theta)$ with the peeling angle is explained by a model in which energy dissipation is due to a hysteretic loss of the work used to stretch the adhesive material when it debonds from the substrate. 


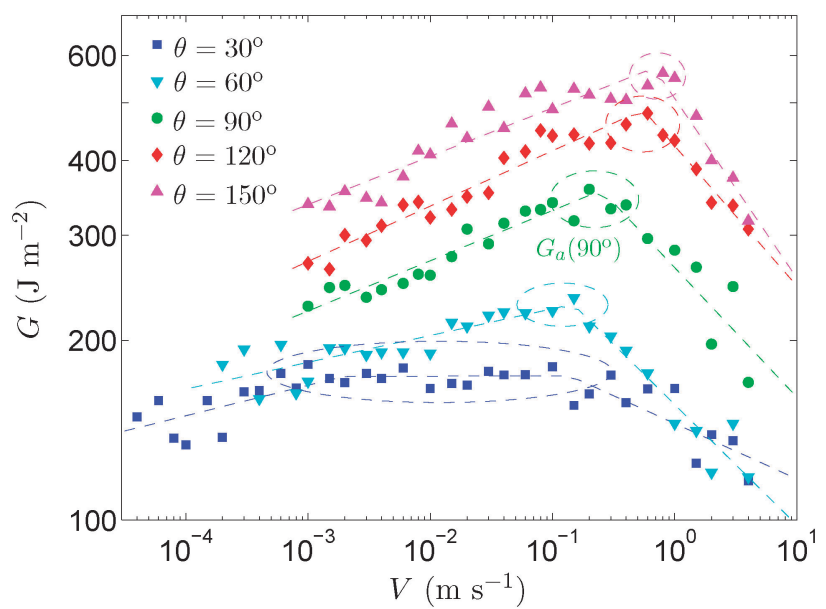

Fig. 7 Energy release rate $G$ as a function of the imposed velocity $V$, for various peeling angles $\theta$. The power law dashed lines are used as guides for the eyes, as well as the circled plateaus of $G(V)$.

As long as $\Gamma$ is an increasing function of $V$, the peeling is steady. Nevertheless, for most pressure sensitive adhesives, it exists a finite range of peeling front velocities $v_{\mathrm{p}} \in\left[V_{\mathrm{a}}, V_{0}\right]$ over which the fracture energy $\Gamma\left(v_{\mathrm{p}}\right)$ is a decreasing function. When the driving velocity $V$ imposed by the operator belongs to this range, the detachment front propagation is potentially unstable and strong periodic oscillations of the front velocity $v_{\mathrm{p}}{ }^{19,21-23}$ and of the peeling force $F^{1-7}$ most often develop. In the present experiments, the onset of the stick-slip instability is indeed observed when $G(V)$ reaches a maximum value (see Fig. 7 and Table 1). As already reported in Dalbe et al., ${ }^{22}$ the threshold velocity $V_{\mathrm{a}}$ above which stick-slip occurs increases with the peeling angle $\theta$. At and beyond this threshold, the peeling force importantly oscillates. In Fig. 7, we still report data for $G(V)$ for driving velocities above the instability onset (which nearly corresponds to the plateaus of $G(V)$, highlighted with dashed ellipses). Since the peeling force strongly oscillates during the corresponding experiments, we actually report the time average of $G(t)$ computed according to eqn (2). In this unstable regime, these data can however not anymore be considered as a measure of the fracture energy of the system associated to a front velocity $V$. We observe that this effective energy release rate decreases rapidly with the driving velocity $V$, except at $30^{\circ}$ where $G$ initially describes a large plateau up to $V \simeq 0.15 \mathrm{~m} \mathrm{~s}^{-1}$ before gently decreasing.
At larger driving velocities, the energy release rate is expected to become an increasing function of $V$ anew and the detachment front dynamics to become stable again, as Barquins and Ciccotti ${ }^{20}$ reported for a very close adhesive-substrate joint (for $\theta=90^{\circ}$ ). Unfortunately, in our experiments, we were only able to reach the beginning of this fast stable peeling branch and furthermore only for $\theta \geq 90^{\circ}$. This is because, for our adhesive-substrate joint, it involves driving velocities out of reach of our experimental setup.

\subsection{The quasi-static approach}

Barquins and Maugis ${ }^{9}$ proposed a quasi-static model to describe the unstable dynamics of the peeling front. In this model, the range of velocities $\left[V_{\mathrm{a}}, V_{0}\right]$ over which $\Gamma\left(v_{\mathrm{p}}\right)$ decreases is forbidden to the detachment front velocity $v_{\mathrm{p}}(t)$, which adjusts on time average to the driving velocity $V$ by alternating between periods of time in the low velocity stable domain, $v_{\mathrm{p}}(t)<V_{\mathrm{a}}$ ("stick" phases), and periods of time in the large velocity stable domain, $v_{\mathrm{p}}(t)>V_{0}$ ("slip" phases).

The key ingredient of this approach is the quasi-static hypothesis, assuming that the front dynamics is still, instantaneously, a solution of the steady peeling equation $G(t)=\Gamma\left(v_{\mathrm{p}}(t)\right)$. This equation was associated with the assumption of discontinuities of $v_{\mathrm{p}}(t)$ when the system is about to enter the forbidden velocity range. At these moments, $v_{\mathrm{p}}(t)$ is assumed to jump instantly from one stable branch of $\Gamma\left(v_{\mathrm{p}}\right)$ to the other at constant energy release rate.

Assuming the ribbon is uniformly stretched, we can relate the peeling force to the overall tape elongation $u$

$$
F=\frac{E e b}{L} u \text {. }
$$

In the peeling geometry considered in this work, where peeling proceeds from a flat surface at nearly constant angle and tape length, the tape elongation verifies

$$
u(t)=u_{0}+\left(V t-\ell_{\mathrm{p}}(t)\right)(1-\cos \theta),
$$

with $u_{0}$ the time averaged elongation, $\theta$ the mean peeling angle and $\ell_{\mathrm{p}}(t)$ the detachment front position along the substrate (see demonstration in ref. 22). From eqn (2), we can also express $u$ as a function of $G$ :

$$
u(t)=L(1-\cos \theta)\left(\sqrt{1+\frac{2 G}{L \mathcal{E}_{\theta}}}-1\right),
$$

Table 1 Values $\Delta \Gamma$ of the fracture energy amplitude that allow to fit the measured periods of the instability (presented in Fig. 3(c)) in the quasi-static regime by the theoretical prediction of eqn (14). We also report $G_{a}-G\left(4 \mathrm{~m} \mathrm{~s}^{-1}\right)$, which is predicted to be a lower bound of $\Delta \Gamma$ and is obtained from independent measurements of the energy release rate $G$ reported in Fig. 7, where $G_{a}$ is the maximum of $G$ in the low velocity stable branch and $4 \mathrm{~m} \mathrm{~s}^{-1}$ is the highest driving velocity accessible with our setup. We also report $V_{a}$, which is the boundary velocity between the low velocity stable domain and the unstable domain, defined as the lowest velocity at which the instability can be observed. The uncertainty in $V_{a}$ comes from the fact there is a bistable domain separating the stable and unstable peeling domains, as described in details in ref. 19

\begin{tabular}{lccccc}
\hline$\theta\left(^{\circ}\right)$ & $\Delta \Gamma\left(\mathrm{J} \mathrm{m}^{-2}\right)$ & $G_{\mathrm{a}}-G\left(4 \mathrm{~m} \mathrm{~s}^{-1}\right)\left(\mathrm{J} \mathrm{m}^{-2}\right)$ & $G_{\mathrm{a}}\left(\mathrm{J} \mathrm{m}^{-2}\right)$ & $V_{\mathrm{a}}\left(\mathrm{m} \mathrm{s}^{-1}\right)$ & $G\left(4 \mathrm{~m} \mathrm{~s}^{-1}\right)\left(\mathrm{J} \mathrm{m}^{-2}\right)$ \\
\hline 30 & $50 \pm 2$ & $60 \pm 20$ & $175 \pm 5$ & $0.007 \pm 0.003$ & $115 \pm 15$ \\
60 & $134 \pm 16$ & $105 \pm 20$ & $225 \pm 10$ & $0.15 \pm 0.05$ & $0.3 \pm 0.1$ \\
90 & $204 \pm 25$ & $175 \pm 40$ & $345 \pm 15$ & $0.4 \pm 0.1$ & $120 \pm 10$ \\
120 & $250 \pm 60$ & $150 \pm 40$ & $560 \pm 20$ & $0.7 \pm 0.3$ & $315 \pm 20$
\end{tabular}


with

$$
\mathcal{E}_{\theta}=\frac{E e}{L}(1-\cos \theta)^{2} .
$$

Then, from eqn (4), (5), and the time derivative of eqn (2), we can write:

$$
\begin{aligned}
\frac{\mathrm{d} G}{\mathrm{~d} t} & =\frac{E e}{L} \dot{u}\left(1-\cos \theta+\frac{u}{L}\right) \\
& =\mathcal{E}_{\theta}\left(V-v_{\mathrm{p}}(t)\right) \sqrt{1+\frac{2 G}{L \mathcal{E}_{\theta}}},
\end{aligned}
$$

with

$$
v_{\mathrm{p}}(t)=\Gamma^{-1}(G(t))
$$

This equation can be integrated in order to predict the duration of the stick and slip periods of the dynamics, leading to:

$$
\begin{gathered}
T_{\text {stick }}=\frac{1}{\mathcal{E}_{\theta}} \int_{G_{0}}^{G_{\mathrm{a}}} \frac{\mathrm{d} G}{\sqrt{1+\frac{2 G}{L \mathcal{E}_{\theta}}}\left(V-\Gamma_{\text {slow }}{ }^{-1}(G)\right)}, \\
T_{\text {slip }}=\frac{1}{\mathcal{E}_{\theta}} \int_{G_{0}}^{G_{\mathrm{a}}} \frac{\mathrm{d} G}{\sqrt{1+\frac{2 G}{L \mathcal{E}_{\theta}}}\left(\Gamma_{\text {fast } \left.^{-1}(G)-V\right)}\right.},
\end{gathered}
$$

with $\Gamma_{\text {slow }}\left(v_{\mathrm{p}}\right)$ and $\Gamma_{\text {fast }}\left(v_{\mathrm{p}}\right)$ the low and large velocity stable branches of $\Gamma\left(v_{\mathrm{p}}\right)$ respectively. $G_{0}$ and $G_{\mathrm{a}}$ are the values of $\Gamma$ at the beginning of the high velocity stable branch $\left(G_{0}=\Gamma\left(V_{0}\right)\right)$ and at the end of the low velocity stable branch $\left(G_{\mathrm{a}}=\Gamma\left(V_{\mathrm{a}}\right)>G_{0}\right)$ of $\Gamma\left(v_{\mathrm{p}}\right)$ respectively.

Our measurements of the energy release rate $G$ presented in Fig. 7 show that at peeling angles $\theta \geq 60^{\circ}$, the term $2 G / L \mathcal{E}_{\theta}$ is always smaller than 0.04 and thus can be neglected in eqn (7) and (8). However, for $\theta=30^{\circ}$, this simplification is not possible $\left(2 G / L \mathcal{E}_{\theta}\right.$ is in the range $\left.0.25-0.40\right)$. Nevertheless, we can approximate the square root in eqn (7) and (8) by its first order Taylor expansion, yielding:

$$
T_{\text {stick }} \simeq \frac{1}{\mathcal{E}_{\theta}}\left(\int_{G_{0}}^{G_{\mathrm{a}}} \frac{\mathrm{d} G}{V-\Gamma_{\text {slow }^{-1}(G)}}-\frac{1}{L \mathcal{E}_{\theta}} \int_{G_{0}}^{G_{\mathrm{a}}} \frac{G \mathrm{~d} G}{V-\Gamma_{\text {slow }}{ }^{-1}(G)}\right),
$$

$$
T_{\text {slip }} \simeq \frac{1}{\mathcal{E}_{\theta}}\left(\int_{G_{0}}^{G_{\mathrm{a}}} \frac{\mathrm{d} G}{\Gamma_{\text {fast }}{ }^{-1}(G)-V}-\frac{1}{L \mathcal{E}_{\theta}} \int_{G_{0}}^{G_{\mathrm{a}}} \frac{G \mathrm{~d} G}{\Gamma_{\text {fast }}{ }^{-1}(G)-V}\right) .
$$

For the tape considered here, $\Gamma_{\text {slow }}\left(v_{\mathrm{p}}\right)$ is very slowly increasing with $v_{\mathrm{p}}$, as shown in Fig. 7. Therefore, provided that $V \in\left[V_{\mathrm{a}}, V_{0}\right]$ is not very close to $V_{\mathrm{a}}, V-\Gamma_{\text {slow }}{ }^{-1}(G) \approx V$ and eqn (9) can be well approximated by

$$
T_{\text {stick }} \simeq \frac{L}{E e(1-\cos \theta)^{2}} \frac{\Delta \Gamma}{V}\left(1-\frac{G_{\mathrm{m}}}{E e(1-\cos \theta)^{2}}\right),
$$

with $\Delta \Gamma=G_{\mathrm{a}}-G_{0}$, the difference between the local extrema of $\Gamma\left(v_{\mathrm{p}}\right)$ and $G_{\mathrm{m}}=\left(G_{\mathrm{a}}+G_{0}\right) / 2$. On the contrary, the fast stable branch $\Gamma_{\text {fast }}\left(v_{\mathrm{p}}\right)$ is expected to be very rapidly increasing ${ }^{9,20}$ (scaling for example as $v_{\mathrm{p}}{ }^{4.5}$ for $3 \mathrm{M}$ Scotch ${ }^{\circledR} 600$ peeled from the commercial roll at $90^{\circ}{ }^{19}$ ). Thus, the peeling point is expected to explore only a thin range of velocities during the slip phase and, provided that $V \in\left[V_{\mathrm{a}}, V_{0}\right]$ is not very close to $V_{0}$, the slip duration (8) can be well approximated by

$$
T_{\text {slip }} \simeq \frac{L}{E e(1-\cos \theta)^{2}} \frac{\Delta \Gamma}{V_{\mathrm{m}}-V}\left(1-\frac{G_{\mathrm{m}}}{E e(1-\cos \theta)^{2}}\right) .
$$

Here $V_{\mathrm{m}}$ is the typical front velocity during the slip phase, expected to be slightly larger than $V_{0}$.

As mentioned above, $G_{\mathrm{m}} /\left(E e(1-\cos \theta)^{2}\right)$ is negligible (smaller than 0.015 ) for $\theta \geq 60^{\circ}$, so, this term can be safely neglected. We will only consider it at $\theta=30^{\circ}$, where it is smaller than (and probably of the order) of 0.17 .

Eqn (11) and (12) predict that the relative duration of the slow phase of the velocity oscillations $T_{\text {stick }}$ compared to the whole period $T_{\mathrm{ss}}=T_{\text {stick }}+T_{\text {slip }}$ approximately scales as

$$
R_{\mathrm{T}}=\frac{T_{\text {stick }}}{T_{\mathrm{ss}}} \simeq 1-\frac{V}{V_{\mathrm{m}}} .
$$

For driving velocities much smaller than $V_{\mathrm{m}}\left(V_{\mathrm{m}}\right.$ being of the order of $V_{0}$ ), the slip duration $T_{\text {slip }}$ is therefore negligible compared to the stick duration $T_{\text {stick }}\left(R_{\mathrm{T}} \sim 1\right)$ and eqn (11) stands as a good approximation of the stick-slip period

$$
T_{\mathrm{ss}}^{(\mathrm{qs})} \simeq \frac{L}{E e(1-\cos \theta)^{2}} \frac{\Delta \Gamma}{V}\left(1-\frac{G_{\mathrm{m}}}{E e(1-\cos \theta)^{2}}\right) .
$$

This quasi-static prediction for the stick-slip instability period that we note $T_{\mathrm{ss}}^{(\mathrm{qs})}$ - has been shown to be in quantitative agreement with experimental measurements of Barquins and Maugis ${ }^{9}$ and Dalbe et al. ${ }^{19}$ for $V<0.6 \mathrm{~m} \mathrm{~s}^{-1}$ and $\theta \simeq 90^{\circ}$ during experiments where peeling proceeds directly from the commercial tape roll $\left(V_{0} \simeq 19 \mathrm{~m} \mathrm{~s}^{-1}\right.$ for $3 \mathrm{M} \mathrm{Scotch}{ }^{\circledR} 600$ at $\theta=90^{\circ}$ studied in ref. 19).

The data reported in the previous section reveals a clear agreement with the quasi-static model for the experiments at low peeling angle or low driving velocities. First, one can note that the instability period is proportional to the peeled tape length in agreement with eqn (14) (Fig. 3). Then, we indeed observe ranges of driving velocity $V$ over which $T_{\mathrm{ss}} / L$ is proportional to $1 / V$ (Fig. $4\left(\mathrm{c}\right.$ )): for $\theta=30^{\circ}$ over two decades of $V$, for $60^{\circ}$ over one decade and a half, and for $90^{\circ}$ over half a decade. Best fits of these data by the theoretical prediction of eqn (14) allow for an estimate, reported in Table 1 , of the parameter $\Delta \Gamma(\theta)$ which is a correction to the primary dependency of the instability period with the peeling angle, $T_{\mathrm{ss}} \propto 1 /(1-\cos \theta)^{2}$. We observe that $\Delta \Gamma(\theta)$ increases with the peeling angle $\theta$. It is important though to notice that at large peeling angles, the instability period is nearly constant in the studied range of driving velocities, in contrast with the prediction of eqn (14). Thus, the value estimated for $\Delta \Gamma\left(120^{\circ}\right)$ from very few data points presents significant uncertainty. Moreover, one can obviously not extract an estimate of $\Delta \Gamma\left(150^{\circ}\right)$ from the data period since no quasi-static regime is observed at all for $\theta=150^{\circ}$. Nevertheless, the fact the peeling is in the inertial regime over the whole explored range of velocity $V$ implies that the quasistatic prediction for the period of the oscillations should be smaller than $5 \pm 1.7 \mathrm{~ms} \mathrm{~m}^{-1}$ (the plateau value corresponding to the inertial regime). This inequality being valid down to the 
smallest studied velocity $V=0.3 \mathrm{~m} \mathrm{~s}^{-1}$, we can estimate an upper bound for $\Delta \Gamma(150)^{\circ}$ using eqn (14).

We can finally compare the values of the parameter $\Delta \Gamma(\theta)$ extracted from the measurements of the instability period to the one obtained by independent direct measurements of the effective fracture energy $\Gamma=G(V)$. In the model prediction given by eqn (14), we have $\Delta \Gamma=G_{\mathrm{a}}-G_{0}$, where we recall that $G_{\mathrm{a}}=G\left(V_{\mathrm{a}}\right)$ and $G_{0}=G\left(V_{0}\right)$ are respectively the local maximum of the fracture energy curve $G(V)$ at the end of the low velocity stable peeling domain and its local minimum at the beginning of the large velocity stable peeling domain. Thus, $G_{\text {a }}$ and $G_{0}$ correspond to fracture energy measured during stable peeling experiments. Unfortunately, as explained previously, in the present experiments, it has not been possible to explore the fast stable branch of the fracture energy, and in particular to precisely identify the local minimum $G_{0}$ of $G(V)$. For that reason, we are only able to estimate an upper bound for $G_{0}$ using the time averaged energy release rate $G\left(4 \mathrm{~m} \mathrm{~s}^{-1}\right)$ measured at the largest velocity accessible by our setup $\left(V=4 \mathrm{~m} \mathrm{~s}^{-1}\right)$. Finally, we compute a lower bound for the theoretical prediction $G_{\mathrm{a}}-G_{0}$ of $\Delta \Gamma$, using the quantity $G_{\mathrm{a}}-G\left(4 \mathrm{~m} \mathrm{~s}^{-1}\right)$. Table 1 reports both $G_{\mathrm{a}}-G\left(4 \mathrm{~m} \mathrm{~s}^{-1}\right)$ and the empirical value of $\Delta \Gamma$ obtained from the best fits by eqn (14) of the period the stick-slip instability. The fact $\Delta \Gamma$ is always larger but of the same order of magnitude than $G_{\mathrm{a}}-G\left(4 \mathrm{~m} \mathrm{~s}^{-1}\right)$ and that they evolve with the peeling angle in a similar manner constitute solid indications that the model we use for the stick-slip period is valid.

\subsection{Limits of the quasi-static approach}

We now discuss the evolution of the relative duration $R_{\mathrm{T}}$ of the slow phase of the velocity oscillations compared to the whole instability period. In the range of driving velocities for which the instability period follows the quasi-static prediction (14), we observe in Fig. 6 that $R_{\mathrm{T}}$ remains typically larger than 0.85 for $\theta=30^{\circ}, 0.75$ for $60^{\circ}$ and 0.7 for $90^{\circ}$. These values reveal that the duration of the fast phase of the oscillation is indeed small relatively to $T_{\mathrm{ss}}$ for $\theta=30^{\circ}$, this approximation getting however less relevant as the peeling angle gets larger. For $\theta=30^{\circ}$, we can also highlight that the decrease of $R_{\mathrm{T}}$ with the driving velocity $V$ is linear as predicted by eqn (13): this allows for an estimate of the typical velocity during the slip period, $V_{\mathrm{m}}\left(\theta=30^{\circ}\right)=29 \pm$ $5 \mathrm{~m} \mathrm{~s}^{-1}$, which is indeed the typical velocity measured during the slip phases for this angle, as shown for example in Fig. 4(a) or Fig. 5. This observation more importantly confirms the relevance of the quasi-static approach for both the slow and fast phases of the oscillations for this angle (over the explored range of driving velocities).

However, for angles larger than $30^{\circ}$, we observe a much faster decrease of $R_{\mathrm{T}}$ with $V$ which does not scale as $R_{\mathrm{T}} \simeq 1-V / V_{\mathrm{m}}$. Such a discrepancy shows that even in the range of driving velocities for which the instability period follows the scaling of eqn (11) for $\theta=60^{\circ}$ and $\theta=90^{\circ}$, the fast phase of the oscillation is affected by dynamical effects (while the slow phase of the oscillation, which dominates the oscillation period, still follows the quasi-static model). The fact the slip period rapidly becomes significant as $V$ increases for these two peeling angles implies that the measurements of the parameter $\Delta \Gamma(\theta)$ are slightly overestimated since they were obtained assuming the slip period to be zero.

It is worth noticing that the quasi-static model of Barquins and Maugis also allows to understand qualitatively why the velocity oscillations amplitude is observed to be nearly independent of the driving velocity for $\theta=30^{\circ}$ with $\Delta v_{\mathrm{p}}=38 \pm 5 \mathrm{~m} \mathrm{~s}^{-1}$. The quasi-static exploration of the fast stable branch of $\Gamma\left(v_{\mathrm{p}}\right)$ assumed by this model imposes that the maximum detachment front velocity during the slip phase is $\Gamma_{\text {fast }}{ }^{-1}\left(G_{\mathrm{a}}\right)$ independently of the driving velocity $V$. It is expected that $\Gamma_{\text {fast }}{ }^{-1}\left(G_{\mathrm{a}}\right)$ and therefore $\Delta v_{\mathrm{p}}$ are only a few tens percent larger than the velocity $V_{0}$ at the beginning of the fast stable branch which is very steep ( $V_{\mathrm{m}}$ being in between). This scenario is consistent with the proximity of the estimates of $\Delta v_{\mathrm{p}}=38 \pm 5 \mathrm{~m} \mathrm{~s}^{-1}$ and of $V_{\mathrm{m}}=29 \pm 5 \mathrm{~m} \mathrm{~s}^{-1}$ obtained for $\theta=30^{\circ}$. Fig. 5 reveals that the constancy of $\Delta v_{\mathrm{p}}$ with $V$ is clearly verified only at $\theta=30^{\circ}$ and that it is not observed at $\theta=60^{\circ}$ and $\theta=90^{\circ}$ even when the quasi-static scaling for the instability period seems relevant. This observation strengthens our previous conclusion that at low driving velocities for $\theta=60^{\circ}$ and $\theta=90^{\circ}$, the quasi-static hypothesis is relevant during the "slow/stick" phase, but already not during the "fast/slip" phase. The latter being predicted by the quasi-static model to be much shorter (at low driving velocity $V$ ), it will naturally be sensitive to inertial effects earlier than the former (the stick phase) when increasing $V$.

Most importantly, we have seen (Fig. 4(c)) that for a given peeling angle, the decrease of the instability period $T_{\mathrm{ss}}$ as $1 / V$ predicted by the quasi-static model seems to saturate at a low value $L / v_{\text {sat }}$ (with $v_{\text {sat }}=220 \pm 70 \mathrm{~m} \mathrm{~s}^{-1}$ ) beyond a given driving velocity $V_{\mathrm{c}}(\theta)$. At saturation, the period per unit length of freestanding tape is thus: $T_{\mathrm{ss}} / L=1 / v_{\mathrm{sat}} \simeq(5 \pm 1.7) \times 10^{-3} \mathrm{~s} \mathrm{~m}^{-1}$. We can first compare this value to the time it takes for a dilatational wave to travel along the peeled tape. From the dilatational wave velocity $c=\sqrt{E b e / \mu} \simeq 1035 \pm 80 \mathrm{~m} \mathrm{~s}^{-1}$, where $\mu=(8.5 \pm 0.8) \times$ $10^{-4} \mathrm{~kg} \mathrm{~m}^{-1}$ is the mass density per unit length of the tape, we find that the travel time per unit length of peeled tape is $1 / c \sim(0.97 \pm 0.08) \times 10^{-3} \mathrm{~s} \mathrm{~m}^{-1}$. This value is significantly smaller but not negligible compared to the saturation value $1 / v_{\text {sat }}$. In the inertial regime, we can therefore expect significant spatial inhomogeneities in longitudinal stretch and force, which will propagate along the free standing tape. At the large driving velocities at which the inertial regime generally occurs, a second dynamical effect will also become important. Indeed, the velocity of the peeled tape close to the peeling front strongly fluctuates in line with the front velocity $v_{\mathrm{p}}(t)$ while a constant velocity $V$ is imposed at the other end of the tape, which implies significant oscillations and inhomogeneities of the kinetic energy of the free standing tape.

When the peeling is stable, at a constant velocity $V$, the change in kinetic energy $U_{\mathrm{k}}$ per unit area $A$ of tape peeled off has an order of magnitude $\mathrm{d} U_{\mathrm{k}} / \mathrm{d} A \simeq \mu V^{2} / 2 b$. In our case, for peeling velocities in the low velocity stable range, we always have $\mu V^{2} / 2 b<10^{-1} \mathrm{~J} \mathrm{~m}^{-2}$ which is three orders of magnitude smaller than the elastic energy release rate measured in this regime (see Fig. 7). Kinetic effects are therefore negligible in 
this regime. However, kinetic energy variations are expected to be significantly higher when the peeling dynamics is unstable, since, in this case, fluctuations of the kinetic energy of the entire free standing tape will occur. In the unstable regime, the amplitude of the tape velocity oscillations is expected to be at least of the order of $V$ (giving $\mathrm{d} U_{\mathrm{k}} \sim \mu L V^{2} / 2$ for the whole free-standing tape) whereas the peeling front moves of $V T_{\mathrm{ss}}$ during a stick-slip cycle (giving $\mathrm{d} A \sim b V T_{\mathrm{ss}}$ ). The associated changes in kinetic energy per unit surface of tape peeled off are therefore typically $\mathrm{d} U_{\mathrm{k}} / \mathrm{d} A \sim \mu V^{2} / 2 b \times L / V T_{\mathrm{ss}}$. Compared to stable peeling, the kinetic energy variation is amplified by a factor $L / V T_{\mathrm{ss}}$ and will be maximum in the inertial regime where it will scale as $\mathrm{d} U_{\mathrm{k}} / \mathrm{d} A \sim \mu V v_{\text {sat }} / 2 b$. For peeling velocities well into the inertial regime $V \sim 3 \mathrm{~m} \mathrm{~s}^{-1}$, we thus expect $\mathrm{d} U_{\mathrm{k}} / \mathrm{d} A$ typically of the order $10 \mathrm{~J} \mathrm{~m}^{-2}$. Although still one order of magnitude smaller than the fracture energies reported in Fig. 7 at low driving velocities, it may now play a significant role in the unstable peeling dynamics. Indeed, recent characterizations of the complex dynamics occurring during a $\operatorname{slip}^{26}$ have shown that the effective fracture energy at high velocity may be of the order of $10 \mathrm{~J} \mathrm{~m}^{-2}$ only. Thus, it might become important to consider the effect of changes in kinetic energy of the peeled tape during unstable peeling. In the following section, we put forward a model which introduces this effect to explain the transition from the "stick-slip" to the "inertial" regime of the dynamical instability.

\section{A dynamical model}

In the pure stick-slip regime described in Section 4.2, an increasing elastic energy is stored in the homogenous stretch of the peeled tape during the stick phase and is then rapidly released during the slip phase. Although in the inertial regime of the instability we can expect inhomogeneities in the stretch of the tape travelling as elastic waves along the tape, we will neglect in the following model their impact on the force distribution along the tape and consider that the applied force can still be expressed using eqn (3). We will however not neglect the changes in kinetic energy that will occur when the peeling front accelerates and decelerates.

\subsection{Ideal case: uniform change in kinetic energy}

From a pedagogical point of view, it is interesting to consider first a simplified situation where a uniform change in velocity occurs along the peeled tape. Let us consider, in the reference frame of the substrate, the additional energy cost to the peeling due to the kinetic energy of the moving tape of length $L$ when the peeling front moves at a velocity $v_{\mathrm{p}}$ while keeping a constant angle $\theta$ with the substrate. The corresponding motion of the free standing tape, here assumed to be rigid, is simply composed of a translation at a velocity $v_{\mathrm{p}}\left(\cos \theta \vec{e}_{x}+\sin \theta \vec{e}_{y}\right)$ in the direction tilted of $\theta$ with respect to the substrate, and of a translation at velocity $-v_{\mathrm{p}} \vec{e}_{x}$ with respect to the substrate (see Fig. $1 ;-v_{\mathrm{p}} \vec{e}_{x}$ is the velocity of the detachment front). Thus, the tape velocity is:

$$
\vec{v}_{\mathrm{t}}=v_{\mathrm{p}}\left[(\cos \theta-1) \vec{e}_{x}+\sin \theta \vec{e}_{y}\right],
$$

and its kinetic energy:

$$
U_{\mathrm{k}}=\frac{1}{2} \mu L \vec{v}_{\mathrm{t}}^{2}=\mu L(1-\cos \theta) v_{\mathrm{p}}^{2},
$$

where, as before, $\mu$ is the mass density per unit length of tape. Assuming that, during a time interval $\mathrm{d} t$, the velocity of the tape varies uniformly when the peeling front velocity $v_{\mathrm{p}}$ changes to $v_{\mathrm{p}}+\mathrm{d} v_{\mathrm{p}}$, and considering that a new portion of tape of area $\mathrm{d} A=b v_{\mathrm{p}} \mathrm{d} t$ has been peeled off and accelerated from zero velocity to $\vec{v}_{\mathrm{t}}$, the change in kinetic energy per unit surface of fracture growth is to first order:

$$
\frac{\mathrm{d} U_{\mathrm{k}}}{\mathrm{d} A}=\frac{2 \mu L}{b}(1-\cos \theta) \dot{v}_{\mathrm{p}}+\frac{\mu}{b}(1-\cos \theta) v_{\mathrm{p}}^{2} .
$$

The first term corresponds to the increase in kinetic energy of the whole tape length, while the second term corresponds to the kinetic energy given to the new portion of peeled tape. The energy balance equation for creating a crack surface $\mathrm{d} A$ becomes:

$$
\Gamma \mathrm{d} A=\mathrm{d}\left(W-U_{\mathrm{e}}-U_{\mathrm{k}}\right),
$$

where $W$ is the work done by the external force $F, U_{\mathrm{e}}$ the elastic energy stored in the tape and $U_{\mathrm{k}}$ its kinetic energy. Thus, one can define a dynamic mechanical energy release rate for peeling the area $\mathrm{d} A$ :

$$
G_{\mathrm{d}}=\frac{F}{b}(1-\cos \theta)+\frac{F^{2}}{2 b^{2} e E}-\frac{\mu(1-\cos \theta)}{b}\left[2 L \dot{v}_{\mathrm{p}}+v_{\mathrm{p}}^{2}\right] .
$$

The second term on the right hand side is less than $1 \%$ of the first term for $\theta>60^{\circ}$ and will be neglected. In the inertial regime, the 4 th rhs term can also be neglected compared to the 3rd one. Indeed, in the inertial regime, where the velocity evolves as a quasi-sinusoidal oscillation of amplitude $V, 2 L \dot{v}_{\mathrm{p}} \sim 2 L V \times\left(2 \pi / T_{\mathrm{ss}}\right)=4 \pi V v_{\mathrm{sat}}$, which is much larger than $v_{\mathrm{p}}^{2} \sim V^{2}$. With these approximations, and assuming that the equilibrium between the dynamical energy release rate $G_{\mathrm{d}}(t)$ and the fracture energy $\Gamma\left(v_{\mathrm{p}}(t)\right)$ is valid instantaneously, we obtain the following dynamical equation, valid in the inertial regime, for the detachment front position:

$$
m_{0} \ddot{\ell}_{\mathrm{p}}+k\left(\ell_{\mathrm{p}}-V t\right)=\frac{b}{(1-\cos \theta)^{2}}\left(\Gamma(V)-\Gamma\left(v_{\mathrm{p}}\right)\right),
$$

with $m_{0}=2 \mu L /(1-\cos \theta), \Gamma(V)=E e(1-\cos \theta) u_{0} / L$ and $k=E e b / L$ the stiffness of the peeled tape. We obtain here an equation that is very similar to the one that was guessed previously ${ }^{22}$ with the added value that we have now an explicit prediction of the effective mass $m_{0}$ that depends on the mass $\mu L$ of the free standing tape and the peeling angle. The caveat of this simple description is that the assumption of a uniform velocity along the free standing tape, used to calculate the kinetic energy, is incompatible with eqn (4) describing the change in stretch that is needed to obtain eqn (19). In order to get a more realistic picture, we will now adapt this simple model to take into account the difference in velocities between both ends of the peeled tape in the calculation of the kinetic energy. 


\subsection{Realistic case: non-uniform change in kinetic energy}

In our experiments, a velocity $V$ is imposed at the pulling end, while the peeling velocity at the front is $v_{\mathrm{p}}$. The distance between the pulling point and the peeling front evolves due to this velocity difference. This effect is taken into account in eqn (4) to estimate the overall tape elongation and thus the associated static force. This equation was derived in ref. 22 using an Eulerian point of view where the evolution of the distance between the motor and the peeling front location was considered, taking into account matter coming in and out at both ends. Equivalently, we could have derived this equation using a Lagrangian point of view by considering, in the reference frame of the substrate, the displacement of the material points at the peeling front and at the pulling end during a time interval $\mathrm{d} t$, knowing the velocities at both ends. The velocity of the material point located at the peeling front can be approximated by eqn (15). This approximation amounts to neglect the additional displacement due to the strain $\varepsilon \approx u / L$ that would give second order corrections. The angle $\theta$ should also be, in principle, a function of time. However, provided that the front oscillations $\ell_{\mathrm{p}}-V t$ are small compared to the tape length $L$ (a few percents in the worst case here), its variations are small (about $2^{\circ}$ in the worst case here, see eqn (12) in ref. 22), and will also give second order corrections that can be neglected. In the experimental configuration studied here, at the pulling end, the velocity of the material point is obtained by replacing $v_{\mathrm{p}}$ by $V$ in eqn (15). The difference in velocity is accommodated by the stretch of the tape. Assuming that it is homogeneous along the peeled tape, the velocity of a material point at a location $s(0<s<L)$ along the tape will simply correspond to a linear interpolation between the velocities at both ends:

$$
\vec{v}_{\mathrm{t}}=v(s)\left[(\cos \theta-1) \vec{e}_{x}+\sin \theta \vec{e}_{y}\right]
$$

where $v(s)=v_{\mathrm{p}}+\left(V-v_{\mathrm{p}}\right) s / L . \dagger$ The kinetic energy of the tape is thus:

$$
\begin{aligned}
U_{\mathrm{k}} & =\int_{0}^{L} \frac{1}{2} \mu \vec{v}_{t}^{2} \mathrm{~d} s, \\
& =\mu L(1-\cos \theta)\left(\frac{v_{\mathrm{p}}^{2}+v_{\mathrm{p}} V+V^{2}}{3}\right) .
\end{aligned}
$$

As already discussed in the previous Section 5.1, we can neglect in the inertial regime the kinetic energy variations due to the new portion of tape peeled off. Thus, the change in kinetic energy per unit surface of fracture growth is to first order:

$$
\frac{\mathrm{d} U_{\mathrm{k}}}{\mathrm{d} A}=\frac{2 \mu L}{b}(1-\cos \theta)\left(\frac{V+2 v_{\mathrm{p}}}{6 v_{\mathrm{p}}}\right) \dot{v}_{\mathrm{p}} .
$$

\footnotetext{
$\dagger$ This relation derives from the following considerations. For a uniformly stretched tape, the elongation $u(s)$ at location $s$ is related to the elongation at $L$ by the relation $u(s)=u(L) \times s / L$ and its variation during a time interval $\mathrm{d} t$ is $\mathrm{d} u(s)=$ $\mathrm{d} u(L) \times s / L$. The value $u(L)$ is given by eqn (4) from which we get $\mathrm{d} u(L)=(V-$ $\left.v_{\mathrm{p}}\right) \mathrm{d} t(1-\cos \theta)$. The same equation can be applied at location $s$ instead of $L$ which gives $\mathrm{d} u(s)=\left(v(s)-v_{\mathrm{p}}\right) \mathrm{d} t(1-\cos \theta)$.
}

The dynamical energy release rate is then:

$$
G_{\mathrm{d}}=\frac{F}{b}(1-\cos \theta)+\frac{F^{2}}{2 b^{2} e E}-\frac{2 \mu L}{b}(1-\cos \theta)\left(\frac{V+2 v_{\mathrm{p}}}{6 v_{\mathrm{p}}}\right) \dot{v}_{\mathrm{p}} .
$$

Neglecting the second rhs term, as justified above, and assuming $G_{\mathrm{d}}(t)=\Gamma\left(v_{\mathrm{p}}\right)$, we obtain the following equation of motion:

$$
m\left(v_{\mathrm{p}}\right) \ddot{\ell}_{\mathrm{p}}+k\left(\ell_{\mathrm{p}}-V t\right)=\frac{b}{(1-\cos \theta)^{2}}\left(\Gamma(V)-\Gamma\left(v_{\mathrm{p}}\right)\right),
$$

where $m\left(v_{\mathrm{p}}\right)=\left(\frac{V+2 v_{\mathrm{p}}}{6 v_{\mathrm{p}}}\right) m_{0}$. The effective mass $m$ is not a constant since it contains an explicit dependency on the instantaneous peeling velocity. However, as we will discuss below, replacing in the inertial factor $m$ the instantaneous velocity $v_{\mathrm{p}}$ by its time averaged value $\left\langle v_{\mathrm{p}}\right\rangle=V$ so that $\langle m\rangle=$ $m_{0} / 2$ provides a very good quantitative description of the oscillation frequency in the inertial regime.

\subsection{Discussion}

We will first discuss the predictions of eqn (19) corresponding to a uniform change in kinetic energy (Section 5.1). For $\theta$ ranging from $30^{\circ}$ to $150^{\circ}$, we have observed experimentally that, in the studied range of driving velocity, the limit cycles of the instability change from stick-slip relaxations to sinusoidal oscillations. In parallel, we note that the geometrical prefactor of the rhs term of eqn (19), $1 /(1-\cos \theta)^{2}$, is reduced by a factor 200. In the limit of large peeling angles, let us consequently neglect this rhs term with respect to the elastic term (2nd term on the lhs of eqn (19)), leading to:

$$
m_{0} \ddot{\ell}_{\mathrm{p}}+k\left(\ell_{\mathrm{p}}-V t\right)=0,
$$

which is a simple equation of a driven harmonic oscillator.

Observation of the experimental limit cycles in the inertial regime (Fig. 4(b)) shows that the detachment front velocity reaches once every cycle an almost zero velocity. Taking this moment as an initial condition to eqn (25) finally yields

$$
v_{\mathrm{p}}=\frac{\mathrm{d} \ell_{\mathrm{p}}}{\mathrm{d} t}=V\left(1-\cos \left(2 \pi \frac{t}{T_{\mathrm{ss}}^{(\mathrm{i})}}\right)\right),
$$

with

$$
T_{\mathrm{ss}}^{(\mathrm{i})}=2 \pi \sqrt{\frac{m_{0}}{k}}=\frac{2 \sqrt{2} \pi}{\sqrt{1-\cos \theta}} \frac{L}{c} .
$$

Solution (26) is consistent with the quasi-sinusoidal shape of the limit cycles observed at large driving velocity and peeling angle (Fig. 4(b)), for which we can in particular verify that the slow and fast phases become of comparable durations in Fig. 6 $\left(R_{\mathrm{T}} \sim 0.5\right)$. The prediction for a peak-to-peak amplitude $\Delta v_{\mathrm{p}}$ scaling as $2 V$ is also consistent with the data reported in Fig. 5 at large driving velocity or peeling angle. Eqn (27) for the oscillation period in the inertial regime predicts values for $T_{\mathrm{ss}} / L$ ranging from $6.3 \times 10^{-3} \mathrm{~s} \mathrm{~m}^{-1}$ to $12.1 \times 10^{-3} \mathrm{~s} \mathrm{~m}^{-1}$ for $\theta$ ranging from $150^{\circ}$ to $60^{\circ}$ which is of the correct order of 


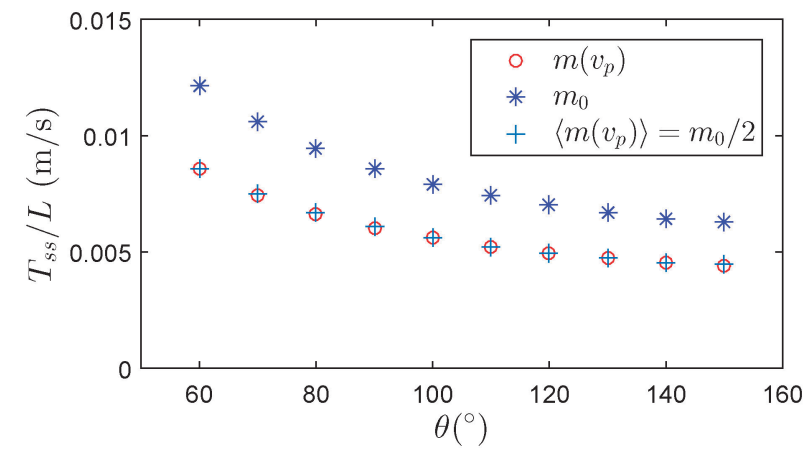

Fig. 8 Predicted values of $T_{s s}^{(i)} / L$ using eqn (27) (inertial factor $m_{0}$ ), a numerical solution of eqn (28) (inertial factor $m\left(v_{p}\right)$ ) or using eqn (29) (approximate inertial factor $\left\langle m\left(v_{p}\right)\right\rangle=m_{0} / 2$ ).

magnitude but is significantly larger than the experimental measurements which are in the range $(5 \pm 1.7) \times 10^{-3} \mathrm{~s} \mathrm{~m}^{-1}$.

Considering now the more realistic approach provided by eqn (24), and again neglecting the right hand side as just above, we get:

$$
m\left(v_{\mathrm{p}}\right) \ddot{\ell}_{\mathrm{p}}+k\left(\ell_{\mathrm{p}}-V t\right)=0 .
$$

We can either solve numerically this equation to obtain the oscillation frequency, or use an approximate version of this equation by replacing $m\left(v_{\mathrm{p}}\right)$ by $\left\langle m\left(v_{\mathrm{p}}\right)\right\rangle=m_{0} / 2$ which leads to a period:

$$
T_{\mathrm{ss}}^{(\mathrm{r})}=2 \pi \sqrt{\frac{\left\langle m\left(v_{\mathrm{p}}\right)\right\rangle}{k}}=\frac{2 \pi}{\sqrt{1-\cos \theta}} \frac{L}{c} .
$$

We find that the numerical integration and the approximate solution give very close values for $T_{\mathrm{ss}} / L$ (Fig. 8). Furthermore, the range of values predicted for $T_{\mathrm{ss}} / L$, from $4.5 \times 10^{-3} \mathrm{~s} \mathrm{~m}^{-1}$ to $8.6 \times 10^{-3} \mathrm{~s} \mathrm{~m}^{-1}$ for $\theta$ ranging from $150^{\circ}$ to $60^{\circ}$, is now in good agreement with the experimental measurements $(5 \pm 1.7) \times$ $10^{-3} \mathrm{~s} \mathrm{~m}^{-1}$ (see also Fig. 3).

The main assumption that lead to the simplified eqn (25) or (28) is that the fracture energy fluctuations can be neglected with respect to kinetic and elastic contributions to the equation of motion at large peeling angles and at large driving velocities. It is worth noting that the same approximate equation could also be relevant for driving velocities (even small!) when an unstable dynamics of the detachment front velocity would imply only weak oscillations of the fracture energy $\Gamma$ which is the case close to maxima or minima of $\Gamma\left(v_{\mathrm{p}}\right)$. This is what happens for instance around the maximum of fracture energy observed in Fig. 7, just at the onset of the unstable range of driving velocity. A plateau of fracture energy has also been reported at high velocities by several authors, ${ }^{8,11}$ when peeling becomes stable again. For both transition regions between stable and unstable peeling, quasi-sinusoidal oscillations of the peeling front dynamics are therefore expected with a characteristic period $T_{\mathrm{ss}}^{(\mathrm{r})}$, identical to the one predicted here. In ref. 23, we have reported the existence of such oscillations at the appearance and disappearance threshold of the stick-slip instability. These observations did not however correspond to steady-state peeling conditions and more detailed characterizations of the transition regions where stick-slip appears and disappears are needed to confirm whether the model presented here would also predict the correct oscillation frequency of the peeling velocity in these regimes.

\subsection{Transition between stick-slip and inertial regimes}

Considering the oscillation period, the transition between the two asymptotic regimes of the unstable peeling will take place when the quasi-static and inertial predictions become comparable, i.e. $T_{\mathrm{ss}}^{(\mathrm{r})}=T_{\mathrm{ss}}^{(\mathrm{qs})}$, which gives

$$
V_{\mathrm{c}}(\theta)=\frac{c \Delta \Gamma(\theta)}{2 \pi E e(1-\cos \theta)^{3 / 2}},
$$

neglecting once again the second rhs term in eqn (2) and thus the second rhs term in eqn (14). This transition velocity estimates when the kinetic energy variations of the free-standing tape become comparable to the variations of the adherence energy during the unstable peeling. Using the values of $\Delta \Gamma(\theta)$ extracted from the fits of the oscillation period (Table 1), we evaluate from eqn (30) that $V_{\mathrm{c}}=[3.5,1.30,0.70,0.47,<0.37] \mathrm{m} \mathrm{s}^{-1}( \pm 20 \%)$ for $\theta=\left[30^{\circ}, 60^{\circ}, 90^{\circ}, 120^{\circ}, 150^{\circ}\right]$.

To summarize our findings, we plot in Fig. 9, the experimental period $T_{\mathrm{ss}}$ of the instability oscillation rescaled by $T_{\mathrm{ss}}^{(\mathrm{r})}=$ $2 \pi L / c \sqrt{1-\cos \theta}$ as a function of the driving velocity rescaled by the characteristic velocity $V / V_{\mathrm{c}}(\theta)$. We show that our model provides a reasonable quantitative prediction for the period of the oscillating peeling dynamics, from the quasi-static regime observed below $V_{c}$, to an inertial regime, where the period saturates theoretically to a low value $T_{\mathrm{ss}}^{(\mathrm{r})}(\theta)$, independent of the driving velocity $V$. Experimentally, a stabilization of the period is indeed observed but with a weak dependence on $V$.

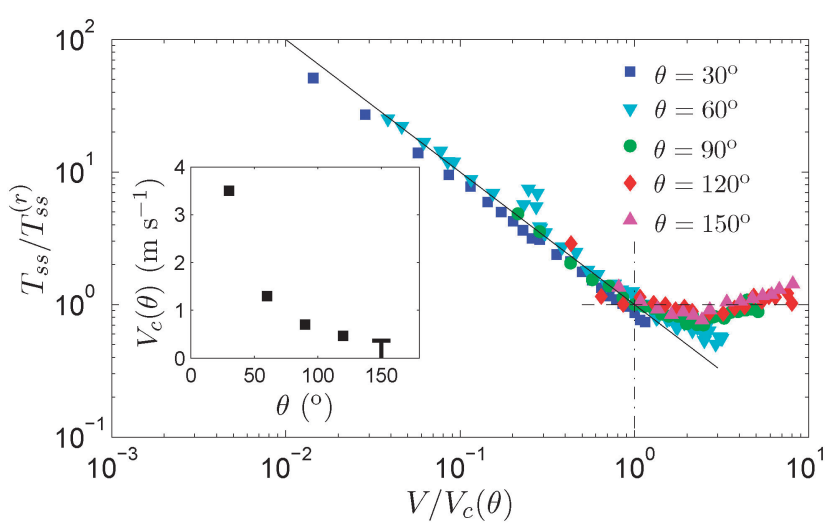

Fig. 9 Period $T_{\text {ss }}$ of the instability oscillation rescaled by $2 \pi L / c \sqrt{1-\cos \theta}$ (corresponding to the prediction of the instability period for the inertial regime $T_{\mathrm{ss}}^{(\mathrm{r})}$ ), as a function of the driving velocity $V$ rescaled by the velocity $V_{c}(\theta)$, separating quasi-static and inertial regimes of the instability. The straight line agrees with the prediction of the quasi-static regime observed below $V_{c}$, while the dashed horizontal line corresponds to the prediction for the inertial regime expected above $V_{c}$. In inset, we plot the evolution of the characteristic velocity $V_{c}$ as a function of the peeling angle $\theta$, obtained from the measurements of the effective fracture energy $\Delta \Gamma(\theta)$ given in Table 1. For $\theta=150^{\circ}$, the upper bound value of $V_{c}$ (at the top of the $T$ symbol) has been used to obtain the collapse in the main figure. 
We have performed a detailed experimental study of the stickslip instability when peeling an adhesive tape from a flat substrate at a constant driving velocity $V$, while maintaining constant both the peeling angle $\theta$ and length $L$ of free standing tape. Thanks to our experimental set-up, we fully characterize the evolution of the period and amplitude of the detachment front oscillations, as function of the control parameters of the peeling $V, L$ and $\theta$. We show that a purely quasi-static "stickslip" model is not enough to describe the evolution of the characteristics of the instability (specifically, the duration and amplitude of the oscillations). Our work allows to identify the kinetic energy variations of the whole moving tape as the inertial effect responsible for the departures of the instability from the traditional "stick-slip" dynamics. Notably, the inertial effect is peeling angle-dependent. Above a characteristic velocity $V_{\mathrm{c}}(\theta)$, related to the effective fracture energy $\Gamma$ of the adhesivesubstrate joint, the period of the oscillations of the instability saturates to a low value that depends very weakly on the driving velocity $V$. In this inertial regime, the velocity oscillations become quasi-sinusoidal (with therefore, slow and fast phases of comparable durations). The model described in this paper may also provide an explanation for the oscillatory peeling motion that was previously observed ${ }^{23}$ at the instability appearance and disappearance velocity thresholds. More experimental work focusing on the onset of the instability will be needed to verify if it is the case.

Finally, further developments of the present model will be necessary to account for a second instability of the peeling front dynamics evidenced in 2010 by Thoroddsen et $a .^{27}$ This instability, involving significantly smaller temporal and spatial scales, may contribute to the characteristic sound of the unstable peeling of PSA. It consists in the regular propagation of transverse dynamic fractures and has been shown ${ }^{26}$ to be the consequence of a high-frequency periodic release of the elastic bending energy of the ribbon concentrated in the vicinity of the peeling front. How this mesoscopic instability interacts with the macroscopic one that was described here remains an open issue.

\section{Acknowledgements}

We thank A. Aubertin, L. Auffray and R. Pidoux for their help in the conception of the experiment, and C. Creton, J.-P. Hulin and B. Saintyves for fruitful scientific discussions. This work has been supported by the French ANR through Grant "StickSlip" No. 12-BS09-014.
References

1 J. L. Gardon, J. Appl. Polym. Sci., 1963, 7, 625.

2 D. H. Kaelble, J. Colloid Sci., 1964, 19, 413.

3 A. N. Gent and R. P. Petrich, Proc. R. Soc. London, Ser. A, 1969, 310, 433-448.

4 D. W. Aubrey, G. N. Welding and T. Wong, J. Appl. Polym. Sci., 1969, 13, 2193.

5 D. W. Aubrey and M. Sherriff, J. Polym. Sci., 1980, 18, 2597.

6 G. Ryschenkow and H. Arribart, J. Adhes., 1996, 58, 143.

7 Y. Yamazaki and A. Toda, Physica D, 2006, 214, 120.

8 M. Barquins, B. Khandani and D. Maugis, C. R. Acad. Sci., Ser. II: Mec., Phys., Chim., Sci. Terre Univers, 1986, 303, 1517.

9 D. Maugis and M. Barquins, in Adhesion 12, ed. K. W. Allen, Elsevier ASP, London, 1988, p. 205.

10 C. Derail, A. Allal, G. Marin and P. Tordjeman, J. Adhes., 1997, 61, 123.

11 C. Derail, A. Allal, G. Marin and P. Tordjeman, J. Adhes., 1998, 68, 203.

12 D. Maugis, J. Mater. Sci., 1985, 20, 3041-3073.

13 P.-G. de Gennes, C. R. Acad. Sci., Ser. II: Mec., Phys., Chim., Sci. Terre Univers, 1988, 307, 1949.

14 P.-G. de Gennes, Langmuir, 1996, 12, 4497-4500.

15 A. N. Gent, Langmuir, 1996, 12, 4492-4496.

16 E. Barthel and C. Fretigny, J. Phys. D: Appl. Phys., 2009, 42, 19.

17 H. Tabuteau, S. Mora, M. Ciccotti, C.-Y. Hui and C. Ligoure, Soft Matter, 2011, 7, 9474-9483.

18 R. Villey, C. Creton, P.-P. Cortet, M.-J. Dalbe, T. Jet, B. Saintyves, S. Santucci, L. Vanel, D. J. Yarusso and M. Ciccotti, Soft Matter, 2015, 11, 3480.

19 M.-J. Dalbe, S. Santucci, P.-P. Cortet and L. Vanel, Soft Matter, 2014, 10, 132.

20 M. Barquins and M. Ciccotti, Int. J. Adhes. Adhes., 1997, 17, 65-68.

21 P.-P. Cortet, M.-J. Dalbe, C. Guerra, C. Cohen, M. Ciccotti, S. Santucci and L. Vanel, Phys. Rev. E: Stat., Nonlinear, Soft Matter Phys., 2013, 87, 022601.

22 M.-J. Dalbe, S. Santucci, L. Vanel and P.-P. Cortet, Soft Matter, 2014, 10, 9637-9643.

23 P.-P. Cortet, M. Ciccotti and L. Vanel, J. Stat. Mech., 2007, P03005.

24 N. Amouroux, J. Petit and L. Léger, Langmuir, 2001, 17, 6510.

25 K. Kendall, J. Phys. D: Appl. Phys., 1975, 8, 1449-1452.

26 M.-J. Dalbe, P.-P. Cortet, M. Ciccotti, L. Vanel and S. Santucci, Phys. Rev. Lett., 2015, 115(12), 128301.

27 S. T. Thoroddsen, H. D. Nguyen, K. Takehara and T. G. Etoh, Phys. Rev. E: Stat., Nonlinear, Soft Matter Phys., 2010, 82(4), 046107. 